DONGMEI YAN, Ph.D. Candidate ${ }^{1}$

(Corresponding author)

E-mail: ydm_1988@163.com

JIANHUA GUO, Ph.D. ${ }^{1}$

E-mail:jg2nh@yahoo.com

${ }^{1}$ Intelligent Transportation System Research Centre

Southeast University

Road of Southeast University 2, Jiangning District,

Nanjing, P. R. China
Intelligent Transport Systems Original Scientific Paper

Submitted: 28 Apr. 2020

Accepted: 26 Mar. 2021

DOI: $10.7307 /$ ptt.v33i5.3586

\title{
A MULTICLASS CUMULATIVE PROSPECT THEORY-BASED STOCHASTIC USER EQUILIBRIUM MODEL WITH PATH CONSTRAINTS IN DEGRADABLE TRANSPORT NETWORKS
}

\begin{abstract}
The limited driving range and the unavailability or insufficiency of battery charging stations cause the socalled range anxiety issue for traffic assignment involving battery electric vehicle (BEV) users. In addition, expected utility theory-based stochastic user equilibrium (EUT-SUE) model generates the perfectly rational issue when the travellers make route choice decisions. To tackle these two problems, this article improves the cumulative prospect theory-based stochastic user equilibrium (CPT-SUE) model in a degradable transport network through incorporating the constraints of multiple user classes and distance limit. In this degradable network, the travellers experience stochastic travel times due to network link capacity degradations. For this improved CPT-SUE model, the equivalent variational inequality (VI) model and associated method of successive averages (MSA) based solution are provided. The improved CPTSUE model is tested and compared with the EUT-SUE model with distance limit, with results showing that the improved CPT-SUE model can handle jointly the range anxiety issue and the perfectly rational issue.
\end{abstract}

\section{KEYWORDS}

stochastic user equilibrium; cumulative prospect theory; expected utility theory; distance limit; variational inequality; method of successive averages.

\section{INTRODUCTION}

Electric vehicles (EVs) are believed to be one of the evidently promising approaches to reduce greenhouse emissions and air pollution. A recent report demonstrated that an $\mathrm{EV}$ can reduce 55 to 60 percent $\mathrm{CO}_{2}$ emission, in comparison with the conventional internal combustion engine (ICE) car in Europe [1]. It is expected that EVs will capture an important market share in the near future with the maturity of EV technology and increased public acceptance. In a recent study, it is predicted that EVs on the U.S. roads will account for 5\% to $14 \%$ of the total number of vehicles by 2030 [2]. Despite the increasing adoptions of EVs, especially battery electric vehicles (BEVs), into the road traffic, the limited driving range (e.g. Pearre et al. [3]) and scarcity of battery charging/swapping stations (e.g. Morrow et al. [4] and Dong et al. [5]) pose a wellknown range anxiety issue [6]. It may be interpreted as travellers afraid of being stranded due to a dead battery [7]. Even though many cities are planning to build and expand the charging infrastructure for $\mathrm{BEVs}$, it is generally acknowledged that range anxiety may still exist in the near future [8].

Traffic assignment is an effective tool for transportation management. The traffic assignment problem accounting for driving range limit of BEVs is initially formulated as user equilibrium (UE) models. In these UE models, the researchers usually convert the driving range limit imposed by range anxiety into a path-level side constraint $[7,9]$. On this basis, the researchers have also investigated design and location for charging station [10-12]. In addition, there have been research studies on the stochastic user equilibrium (SUE) with distance limit of BEVs [13]. Generally speaking, traditional logit-based SUE models (e.g. Maher [14] and Yang et al. [15]) and its extended models (e.g. C-logit stochastic user equilibrium model [16], the cross-nested logit stochastic user equilibrium [17]) in the literature are based on expected utility theory (EUT). 
The EUT has a main assumption, that is, travellers are absolutely rational when making route choice decisions [9]. More specifically, the travellers seek the maximum perceived expected utility by choosing a path with the least expected costs (e.g. travel time) [18]. Therefore, these SUE models with distance limit often have perfectly rational issues. In addition, the aforementioned range anxiety issue for BEVs will hardly be eliminated in the near future [8]. Thus, the range anxiety issue and perfectly rational issue are critical problems to be addressed in BEV-related studies.

The traditional SUE models involving BEVs suffer from the perfectly rational issue and range anxiety issue. At present, the research to jointly solve these two issues is insufficient. Thus, the objective of this paper is to improve the cumulative prospect theory-based stochastic user equilibrium (CPT-SUE) model by incorporating the constraints of multiple user classes and distance limit to jointly deal with the range anxiety issue and perfectly rational issue. For the improved CPT-SUE model, we constructed an equivalent variational inequality (VI) model and proved the existence and equivalence of the model solution. Furthermore, numerical investigations are conducted to compare the path flows by the improved CPT-SUE model and the expected utility theory-based stochastic user equilibrium (EUT-SUE) model with distance limit, together with the analysis of influence of distance limit parameter on path flows of gasoline vehicle $(\mathrm{GV})$ and BEV.

The rest of this paper is organised as follows. Section 2 provides a literature overview of traffic assignment studies involving range anxiety and perfectly rational issues in assignment. Section 3 revisits link and path travel time distributions and cumulative prospect theory. Section 4 formulates the improved CPT-SUE model as an equivalent VI model. Furthermore, the equivalency and existence of model solution are also proved. Section 5 provides a numerical example to demonstrate the performance of the improved CPT-SUE model. Lastly, Section 6 gives the conclusions of the paper.

\section{LITERATURE REVIEW}

The common modelling approach of network flow pattern is the UE model. The UE model considering the distance limit of BEVs has attracted an increasing attention. For instance, Jiang et al. proposed a path-constrained traffic assignment model with distance limit [7], which defines the path flow to be zero if the path length exceeds the distance limit. Jiang et al. further extended the model in [7] to develop a network equilibrium model with distance limit of BEVs [19]. The model in [19] evaluates the impacts of the distance limit on destination, route, and parking choice behaviours under equilibrium conditions. Jiang and Xie formulated a network equilibrium problem with mode and route choice and analysed how the operation cost and distance limit affect vehicle and route choices decisions of the travellers [9]. On this basis, a series of other studies are also conducted. For example, the traffic assignment problem involving flow-dependent electricity consumption for BEVs $[8,10,20]$, the traffic assignment problem considering BEVs recharging [21-24], and the BEV touring, routing and scheduling problems [25-27].

The above studies are all extended versions of the UE model. The UE model assumes that all travellers have perfect information regarding travel time over the entire network. This assumption may not be reasonable. Researchers have relaxed the aforementioned assumption by incorporating more realism into the traditional UE model, and then SUE model was proposed. At present, only a limited number of studies have focused on SUE with distance limit. Jing et al. proposed a SUE model with distance limit [28]. On this basis, Jing et al. proposed a bi-level public charging location model considering BEV range limits and the SUE principle [13]. Ma et al. proposed a SUE model for BEVs considering environmental costs [29].

These three aforementioned SUE models with distance limit are all based on expected utility theory (EUT). The EUT is based on the assumption that travellers are perfectly rational when making route choice decisions. In other words, these models have a perfectly rational issue. To tackle this problem, the researchers have considered embedding the cumulative prospect theory (CPT) (e.g. Tversky and Kahneman [30]) in the traffic assignment model that does not include the distance limit of BEVs. For example, Connors and Sumalee presented a CPTbased user equilibrium (CPT-UE) model with uncertain travel time to study route choice behaviour of travellers [31]. Lou et al. proposed a bounded rational user equilibrium (BRUE) model to investigate the congestion pricing [32]. However, these two studies relied on exogenous inputs of reference points, and no guidance was provided on how to 
derive these values. $\mathrm{Xu}$ et al. extended these two models to consider endogenous reference point (RP) in a multiclass CPT-UE model for stochastic networks [33], which is more competent to predict the long-run user equilibrium flow pattern. Wang and $\mathrm{Xu}$ further encapsulated endogenous RP in [33] into a CPT-UE model [34]. In particular, they considered a degradable transport network caused by inevitable natural disasters and traffic accidents. Wang and Sun extended the model in [34] to embrace stochastic perception error (SPE) in route choice decision process of travellers [35]. Yang and Jiang developed a CPT-SUE model instead of the CPT-UE model by relaxing the assumption of the UE model [36]. To the best knowledge of the authors, there is insufficient study on applying CPT to describe the route choice behaviour of travellers in the SUE model with distance limit. To this end, we improved the CPT-SUE model through incorporating the constraints of multiple user classes (i.e., gasoline vehicles (GVs) and BEVs) and distance limit of BEVs to handle the range anxiety issue and the perfectly rational issue jointly.

\section{CALCULATION OF CUMULATIVE PROSPECT VALUE}

To simplify the complexity of the improved CPT-SUE model, a series of assumptions are proposed based on the research of Jiang et al [19].

- GV users and BEV users are considered for the travel demand population.

- Total travel demand for each type of vehicle at each origin is fixed.

- GV users and BEV users spend the same amount of time on the same path.

- All BEVs are assumed to be fully charged at origins.

\subsection{Path travel time distribution}

In this study, the Bureau of Public Roads (BPR) function (see [37]) is adopted as the following:

$T_{a}\left(x_{a}, C_{a}\right)=t_{a}^{0}\left[1+\alpha\left(\frac{x_{a}}{C_{a}}\right)^{\beta}\right] \forall a$

where $a$ is a specific link, $t_{a}^{0}$ is free-flow travel time on link $a, x_{a}$ is flow on link $a, C_{a}$ is capacity on link $a, \alpha$ and $\beta$ are deterministic parameters.

In a degradable transport network, link capacity is stochastically changing due to major events, such as earthquakes and traffic incidents. Therefore, the link capacity is a random variable subject to stochastic degradation. It is assumed that the link capacity follows a uniform distribution [38]. The upper bound of the uniform distribution interval length of the link capability is the design capability $\bar{c}$, while lower bound is a fraction $\theta_{a}$ of the design capacity. According to Lo and Siu [38], the mean and variance of path travel time distribution are obtained as below.

$$
\begin{aligned}
& E\left(T_{k, i}^{w}\right)=\sum_{a}\left\{\delta_{a, k}^{w}\left[t_{a}^{0}+\alpha t_{a}^{0} x_{a}^{\beta} \frac{1-\theta_{a}^{1-\beta}}{\bar{c}_{a}^{\beta}\left(1-\theta_{a}\right)(1-\beta)}\right]\right\} \\
& \quad \forall w, k, i \\
& \operatorname{var}\left(T_{k, i}^{w}\right) \\
& =\sum_{a}\left\{\delta_{a, k}^{w} \alpha^{2}\left(t_{a}^{0}\right)^{2} x_{a}^{2 \beta}\left[\frac{1-\theta_{a}^{1-2 \beta}}{\bar{c}_{a}^{2 \beta}\left(1-\theta_{a}\right)(1-2 \beta)}-\left(\frac{1-\theta_{a}^{1-\beta}}{\bar{c}_{a}^{\beta}\left(1-\theta_{a}\right)(1-\beta)}\right)^{2}\right]\right\} \\
& \forall w, k, i
\end{aligned}
$$

where $w$ is a specific O-D pair, $k$ is a specific path, $T_{k, i}^{w}$ is the random variable of travel time of user class $i$ on path $k$ between O-D pair $w, \bar{c}_{a}$ is design capability on link $a, \theta_{a}$ is worst-degraded coefficient on link $a, \delta_{a, k}^{w}$ is the relationship between path $k$ and link $a$. Note that $\delta_{a, k}^{w}=1$ if a is contained by $k$, otherwise $\delta_{a, k}^{w}=0$.

\subsection{Reference point}

The reference point (RP) is the turning point of risk attitudes of travellers. More specifically, when the path travel time is smaller than the RP, the travellers may be risk averse. In contrast, the travellers could be risk seeking when the path travel time is larger than the RP. The endogenous RP proposed by $\mathrm{Xu}$ et al. [33] is adopted in this article. As noted, we know that link capacity degradations cause the variation of link and path travel time. Travellers, therefore, do not know the exact time to arrive at their destination because of random variation in path travel time. Most travellers will choose to depart earlier or increase travel time margins based on average travel time to avoid late arrivals. Thus, the travel time budget can be defined as the summation of the average travel time and the travel time margin. The travel time budget can be expressed as follows:

$b_{k, i}^{w}=E\left(T_{k, i}^{w}\right)+\lambda_{i} \sigma_{k, i}^{w} \quad \forall w, k, i$

where $b_{k, i}^{w}$ is travel time budget of user class $i$ on path $k$ between the O-D pair w, $\sigma_{k, i}^{w}$ is the standard deviation of the random variable $T_{k, i}^{w}, \lambda_{i}$ is a parameter related to the user class $i$ on-time arrival probability $\rho_{i}$, written as:

$P\left\{T_{k, i}^{w} \leq b_{k, i}^{w}=E\left(T_{k, i}^{w}\right)+\lambda_{i} \sigma_{k, i}^{w}\right\}=\rho_{i} \quad \forall w, k, i$ 
where $\rho_{i}$ is on-time arrival probability within the travel time budget. Equation 6 then can be derived from Equation 5 and is given by:

$$
b_{k, i}^{w}=E\left(T_{k, i}^{w}\right)+\sigma_{k, i}^{w} \Phi^{-1}\left(\rho_{i}\right) \quad \forall w, k, i
$$

where $\Phi^{-1}$ is the inverse function of the standard normal cumulative distribution function. In this article, the minimum travel time budget is regarded as endogenous RP, that is

$u_{0, i}^{w}=\min _{k}\left\{b_{k, i}^{w}\right\} \quad \forall w, i$

where $u_{0, i}^{w}$ is RP of user class $i$ between O-D pair $w$.

\subsection{Cumulative prospect theory}

The cumulative prospect theory (CPT) provides a well-supported descriptive paradigm for decision making of individuals under risk or uncertainty [33]. The key elements of CPT are the value function and the probability weight function. CPT has three features that have been observed in numerous behavioural experiments: (i) People tend to identify gains or losses relative to a reference point before making a choice [39]. (ii) People are usually more concerned about potential losses than potential gains. At the same time, they are risk aversion over gains and risk seeking over losses [40]. (iii) People tend to overweigh small probability events and underweigh moderate and high probability events [41].

1) Value function

In this section, we use the value function instead of utility function to capture individual's risk attitude. Value function exhibits concave for the gain, convex for the loss, and its curve is steeper in the loss region than the gain region. According to $\mathrm{Xu}$ et al. [33], value function is described as below.

$g\left(T_{k, i}^{w}\right)=\left\{\begin{array}{l}\left(u_{0, i}^{w}-T_{k, i}^{w}\right)^{\vartheta}, T_{k, i}^{w} \leq u_{0, i}^{w} \\ -\mu\left(T_{k, i}^{w}-u_{0, i}^{w}\right)^{\eta}, T_{k, i}^{w}>u_{0, i}^{w}\end{array} \forall w, k, i\right.$

where $\vartheta$ and $\eta$ are the degree of diminishing sensitivity of value function, $0<\vartheta, \eta<1, \mu$ is loss-aversion coefficient, $\mu \geq 1$.

2) Probability weighting function

It is assumed that the travellers make decisions based on perceived probabilities, which relaxes the assumption that decision makers can act rationally with perfect information about all possible probabilities. The probability weighting function proposed by Prelec [42] is adopted in this paper as shown below.

$\omega(p)=\exp \left[-(-\ln p)^{\gamma}\right] \quad 0<\gamma<1$ where $\omega(p)$ is perceived weight probability of an event, $p$ is actual probability of an event, $\gamma$ is the degree of distortion of the probability judgment in the decision process, $0<\gamma<1$.

3) Cumulative prospect value

The cumulative prospect value (CPV) is calculated by the value function and the probability weight function. According to Zhang et al [43], the CPV perceived by the user class $i$ on path $k$ between the O-D pair $w$ can be described as follows:

$$
\begin{aligned}
v_{k, i}^{w}= & \int_{\substack{u_{k, i}^{w} \\
u_{0, i}^{w}}}^{u_{w}^{w}} \frac{d \omega^{+}\left[F\left(T_{k, i}^{w}\right)\right]}{d T_{k, i}^{w}} g\left(T_{k, i}^{w}\right) d T_{k, i}^{w} \\
& +\int_{\substack{u_{k, i}^{w} \\
u_{0, i}^{w}}}^{w}-\frac{d \omega^{-}\left[1-F\left(T_{k, i}^{w}\right)\right]}{d T_{k, i}^{w}} g\left(T_{k, i}^{w}\right) d T_{k, i}^{w} \\
& \forall w, k, i
\end{aligned}
$$

where $F\left(T_{k, i}^{w}\right)$ is distribution function of $T_{k, i}^{w}, \omega^{+}$ and $\omega^{-}$are gain area and loss area of probability weighting function, respectively, $\underline{u}_{k, i}^{w}$ and $\vec{u}_{k, i}^{w}$ are lower bound and upper bound of the path travel time of user class $i$ on path $k$ between the O-D pair $w$, respectively.

In this paper, the former is assumed to be freeflow travel time. The latter is considered as below.

$\bar{u}_{k, i}^{w}=E\left(T_{k, i}^{w}\right)+3 \sqrt{\operatorname{var}\left(T_{k, i}^{w}\right)} \quad \forall w, k, i$

\section{STUDY MODEL}

\subsection{The improved CPT-SUE model}

In this paper, the travellers choose the path based on path prospect instead of path travel cost. The path prospect $V_{k, i}^{w}$ can be regarded as a random variable distributed in the overall travellers, which can be expressed as follows:

$V_{k, i}^{w}=v_{k, i}^{w}+\varepsilon_{k, i}^{w} \quad \forall w, k, i$

where $v_{k, i}^{w}$ is deterministic part, $\varepsilon_{k, i}^{w}$ is random error.

It is assumed that the random error terms are independently and identically distributed (IID) Gumbel variates. The probability that user class $i$ chooses path $k$ can be exhibited as follows:

$P_{k, i}^{w}=\frac{\exp \left(\theta_{i} v_{k, i}^{w}\right)}{\sum_{k} \exp \left(\theta_{i} v_{k, i}^{w}\right)} \forall w, k, i$

where $\theta_{i}$ is familiarity of user class $i$ with the road network conditions.

According to SUE assignment theory, the equilibrium conditions can be expressed as follows: 


$$
\begin{aligned}
& f_{k, i}^{w}=q_{i}^{w} P_{k, i}^{w} \quad \forall w, k, i \\
& \text { s.t. } \sum_{k} f_{k, i}^{w}=q_{i}^{w} \quad \forall w, k, i \\
& \left(D_{i}-l_{k}^{w}\right) f_{k, i}^{w} \geq 0 \quad \forall w, k, i \\
& x_{a, i}=\sum_{w} \sum_{k} f_{k, i}^{w} \delta_{a, k}^{w} \quad \forall a, i \\
& x_{a}=\sum_{i} x_{a, i} \quad \forall a \\
& f_{k, i}^{w} \geq 0 \quad \forall w, k, i
\end{aligned}
$$

where $q_{i}^{w}$ is demand of user class $i$ between the O-D pair $w, f_{k, i}^{w}$ is path flow of user class $i$ on path $k$ between the O-D pair $w, D_{i}$ is distance limit of the user class $i, l_{k}^{w}$ is the length of path $k$ between the O-D pair $w, x_{a}$ is flow on link $a, x_{a, i}$ is flow of user class $i$ on link $a$.

The satisfaction is the expectation of the maximum utility alternative. In this paper, satisfaction is the expectation of the maximum CPV. The satisfaction can also be expressed as a function of the vector of measured CPV, that is:

$$
S\left(v_{k, i}^{w}\right)=E\left(\max _{k} V_{k, i}^{w}\right) \forall w, k, i
$$

It is noted that the partial derivative of the satisfaction function of CPV of a path is equal to the choice probability of that path, that is:

$$
\frac{\partial S(\mathbf{V})}{\partial V_{k}}=P_{k}(\mathbf{V}) \quad \forall k
$$

where $\mathbf{V}$ is a vector with $v_{k, i}^{w}$ as an element.

According to Sheffi [44], the satisfaction function of the route choice problem is defined as follows:

$$
S\left(v_{k, i}^{w}\right)=\frac{1}{\theta_{i}} \ln \sum_{k} \exp \left(\theta_{i} v_{k, i}^{w}\right) \quad \forall w, k, i
$$

\subsection{The equivalent VI model}

The improved CPT-SUE model is formulated as the following equivalent VI model:

$$
\sum_{w} \sum_{k} \sum_{i}\left[S\left(v_{k, i}^{w}\right)+\frac{1}{\theta_{i}}\left(\ln f_{k, i}^{w^{*}}-\ln q_{i}^{w}\right)-v_{k, i}^{w}\right] \cdot\left(f_{k, i}^{w}-f_{k, i}^{w^{*}}\right) \geq 0
$$

For the proposed VI model, we next prove the equivalence and existence of the model solution between the improved CPT-SUE model and the proposed VI model.

1) The equivalency of model solution

Proposition 1: The VI model is equivalent to the improved CPT-SUE model.

Proof: According to the VI theorem, the VI mod$\mathrm{el}$ is equivalent to the following complementary relaxation conditions:

$$
\begin{aligned}
& {\left[S\left(v_{k, i}^{w}\right)+\frac{1}{\theta_{i}}\left(\ln f_{k, i}^{w *}-\ln q_{i}^{w}\right)-v_{k, i}^{w}\right] \cdot f_{k, i}^{w *}=0 \quad \forall w, k, i} \\
& {\left[S\left(v_{k, i}^{w}\right)+\frac{1}{\theta_{i}}\left(\ln f_{k, i}^{w *}-\ln q_{i}^{w}\right)-v_{k, i}^{w}\right] \geq 0 \quad \forall w, k, i} \\
& f_{k, i}^{w *} \geq 0 \quad \forall w, k, i \\
& \quad \text { When } f_{k, i}^{w}>0, \text { we have } \\
& S\left(v_{k, i}^{w}\right)+\frac{1}{\theta_{i}}\left(\ln f_{k, i}^{w^{*} *}-\ln q_{i}^{w}\right)-v_{k, i}^{w}=0 \quad \forall w, k, i
\end{aligned}
$$

Substituting Equation 22 by Equation 27, we have

$f_{k, i}^{w^{*}}=q_{i}^{w} \cdot \frac{\exp \left(\theta_{i} v_{k, i}^{w}\right)}{\sum_{k} \exp \left(\theta_{i} \mathcal{V}_{k, i}^{w}\right)} \forall w, k, i$

Obviously, Equation 28 is equivalent to the SUE condition. Thus, it is proved that the solution of VI model is equivalent to the solution of the improved CPT-SUE model.

2) The existence of model solution

Proposition 2: The VI model has at least one solution.

Proof: Let

$F_{k, i}^{w^{*}}(f)=S\left(v_{k, i}^{w}\right)+\frac{1}{\theta_{i}}\left(\ln f_{k, i}^{w^{*}}-\ln q_{i}^{w}\right)-v_{k, i}^{w}$,

and $\boldsymbol{F}(f)$ represents a vector with $F_{k, i}^{w}(f)$ as an element. The distribution function of link travel time is assumed to be a continuous function of the link flow. According to Equations 4-7, it is easy to know that RP is a continuous function of the path flow, so $\boldsymbol{F}(\boldsymbol{f})$ is also a continuous function of the path flow. According to constrains 15-19, it is certainly true that the feasible path flow set is a compact convex set. Based on the VI theorem, the solution of Equation 23 exists.

\subsection{MSA-based solution to the VI model}

The MSA is a popular algorithm for solving stochastic user equilibrium model because of its simplicity and ease of engineering implementation. Based on this, we choose the MSA algorithm to solve the equivalent VI model. The steps of applying the MSA algorithm to solve the VI model are presented below.

Step 0: Parameter settings. Determine feasible path set. The distance limit of BEVs is set to a certain value and the distance limit of the GV is infinity. The convergence tolerance $\varepsilon$ and other parameters are given predetermined values.

Step 1: Initialisation. Calculate travel time budget $\quad\left\{\mathbf{b}^{(\mathbf{1})}=\left(b_{k, i}^{w(1)}\right), \quad \forall w, k, i\right\} \quad$ and $\mathrm{CPV}$ $\left\{\mathbf{v}^{(\mathbf{1})}=\left(v_{k, i}^{w(1)}\right), \forall w, k, i\right\}$ based on zero flow. Calculate 
initial path flow $\left\{\mathbf{f}^{(\mathbf{1})}=\left(f_{k, i}^{w(1)}\right), \forall w, k, i\right\}$ based on Equation 14, and then yield a set of $\left\{\mathbf{x}^{(\mathbf{1})}=\left(x_{a}^{(1)}\right), \forall a\right\}$. Set iteration counter $n=1$.

Step 2: Update. Update travel time budget and CPV according to Equation 4 and Equation 10.

Step 3: Direction finding. According to Equation 14, calculate the auxiliary path flow $\left\{\mathbf{g}^{(\mathbf{n})}=\left(g_{k, i}^{w(n)}\right), \forall w, k, i\right\}$ based on updated CPV.

Step 4: Move. Update the path flow as $\mathbf{f}^{(\mathbf{n}+\mathbf{1})}=\mathbf{f}^{(\mathbf{n})}+(1 / \mathrm{n})\left(\mathbf{g}^{(\mathbf{n})}-\mathbf{f}^{(\mathbf{n})}\right)$.

Step 5: Convergence test. If $\left\|\mathbf{f}^{(\mathbf{n}+\mathbf{1})}-\mathbf{f}^{\mathbf{f}(\mathbf{n})}\right\| /\left\|\mathbf{f}^{(\mathbf{n})}\right\| \leq \varepsilon$, then stop. If not, set $n=n+1$ and go to Step 2 .

\section{NUMERICAL EXAMPLE}

\subsection{Preliminary}

The Nguyen-Dupuis network is selected for testing the purpose of this paper. The Nguyen-Dupuis network consists of 4 O-D pairs, 13 nodes, and 19 links, as shown in Figure 1. For each O-D pair, the travel demand is $q_{1-2}=660, q_{1-3}=495, q_{4-2}=412.5$, and $q_{4-3}=495$, respectively. The coefficients of the BPR function are set to $\alpha=0.15$ and $\beta=4$. The parameters of the value function are assumed to be $\vartheta=\eta=0.88$ and $\mu=2.25$. The parameter of probability weighting function is considered as $\gamma=0.74$. For the GV and BEV users, the on-time arrival probability is assumed to be $\rho=[0.7,0.8]$ and the dispersion parameter (i.e., familiarity of travellers with the road network conditions) is set to $\theta=[0.3,0.5]$. In addition, the market share of $\mathrm{GV}$ and $\mathrm{BEV}$ is set to $\tau=[0.7,0.3]$. The distance limit of $\mathrm{BEV}$ is set to $D_{2}=40$. The iteration accuracy is defined as $\varepsilon=0.0001$.

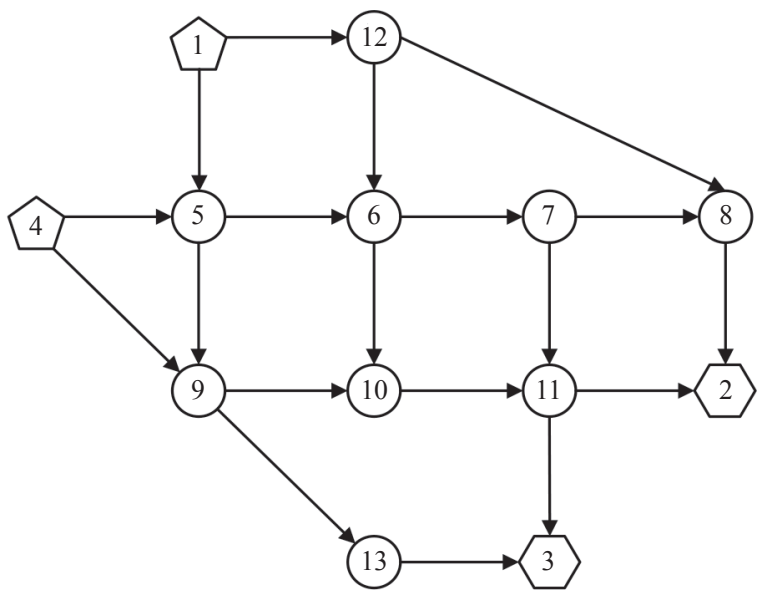

Origin node $\bigcirc$ Destination node $\bigcirc$ Ordinary node

Figure 1 - Nguyen-Dupuis network

Free-flow travel time, capacity (The values in Table 1 are adopted from $\mathrm{Xu}$ et al. [33]) and the worst-degraded coefficient $\theta_{a}$ for each link on the network is also shown in Table 1. The path composition and path length are exhibited in Table 2 (The values in the Table 2 are adopted from Jiang and Xie [9]).

\subsection{Path flow comparison}

The variational inequality model and the corresponding MSA based solution algorithm are coded in MATLAB 2018a. The network equilibrium solutions are shown in Table 3 and Table 4.

On one hand, in Table 3 and Table 4, it can be seen that the path flows assigned by the improved CPTSUE model is significantly different from path flows

Table 1-Link characteristics

\begin{tabular}{||c|c|c|c|c||c|c|c|c|c||}
\hline No. & Link & $t_{a}^{0}$ & $\bar{c}_{a}$ & $\theta_{a}$ & No. & Link & $t_{a}^{0}$ & $\bar{c}_{a}$ & $\theta_{a}$ \\
\hline \hline 1 & $1-5$ & 7 & 300 & 0.8 & 11 & $8-2$ & 9 & 500 & 0.7 \\
\hline 2 & $1-12$ & 9 & 200 & 0.8 & 12 & $9-10$ & 10 & 550 & 0.6 \\
\hline 3 & $4-5$ & 9 & 200 & 0.7 & 13 & $9-13$ & 9 & 200 & 0.8 \\
\hline 4 & $4-9$ & 12 & 200 & 0.8 & 14 & $10-11$ & 6 & 400 & 0.7 \\
\hline 5 & $5-6$ & 3 & 350 & 0.6 & 15 & $11-2$ & 9 & 300 & 0.7 \\
\hline 6 & $5-9$ & 9 & 400 & 0.6 & 16 & $11-3$ & 8 & 300 & 0.6 \\
\hline 7 & $6-7$ & 5 & 500 & 0.7 & 17 & $12-6$ & 7 & 200 & 0.8 \\
\hline 8 & $6-10$ & 13 & 250 & 0.8 & 18 & $12-8$ & 14 & 300 & 0.7 \\
\hline 9 & $7-8$ & 5 & 250 & 0.7 & 19 & $13-3$ & 11 & 200 & 0.7 \\
\hline 10 & $7-11$ & 9 & 300 & 0.7 & & & & \\
\hline
\end{tabular}


Yan D, Guo J. A Multiclass Cumulative Prospect Theory-Based Stochastic User Equilibrium Model with Path Constraints in...

Table 2 - Path composition and length

\begin{tabular}{|c|c|c|c|c|c|c|c|}
\hline O-D & Path & Node sequence & Path length & O-D & Path & Node sequence & Path length \\
\hline \multirow{8}{*}{$1-2$} & 1 & $1-12-8-2$ & 32 & \multirow{8}{*}{$1-3$} & 9 & $1-5-9-13-3$ & 36 \\
\hline & 2 & $1-5-6-7-8-2$ & 29 & & 10 & $1-5-6-7-11-3$ & 32 \\
\hline & 3 & $1-5-6-7-11-2$ & 33 & & 11 & $1-5-6-10-11-3$ & 37 \\
\hline & 4 & $1-5-6-10-11-2$ & 38 & & 12 & $1-5-9-10-11-3$ & 40 \\
\hline & 5 & $1-5-9-10-11-2$ & 41 & & 13 & $1-12-6-7-11-3$ & 38 \\
\hline & 6 & $1-12-6-7-8-2$ & 35 & & 14 & $1-12-6-10-11-3$ & 43 \\
\hline & 7 & $1-12-6-7-11-2$ & 39 & & -- & -- & -- \\
\hline & 8 & $1-12-6-10-11-2$ & 44 & & -- & -- & -- \\
\hline \multirow{6}{*}{$4-2$} & 15 & $4-9-10-11-2$ & 37 & \multirow{6}{*}{$4-3$} & 20 & $4-9-13-3$ & 32 \\
\hline & 16 & $4-5-6-7-8-2$ & 31 & & 21 & $4-9-10-11-3$ & 36 \\
\hline & 17 & $4-5-6-7-11-2$ & 35 & & 22 & $4-5-9-13-3$ & 38 \\
\hline & 18 & $4-5-6-10-11-2$ & 40 & & 23 & $4-5-6-7-11-3$ & 34 \\
\hline & 19 & $4-5-9-10-11-2$ & 43 & & 24 & $4-5-6-10-11-3$ & 39 \\
\hline & -- & -- & -- & & 25 & $4-5-9-10-11-3$ & 42 \\
\hline
\end{tabular}

Table 3 - The network equilibrium solutions calculated by the improved CPT-SUE model

\begin{tabular}{|c|c|c|c|c|c|c|c|}
\hline O-D & Path & GV flow & BEV flow & CPV (GV) & CPV (BEV) & Mean path time & Path time st. dev. \\
\hline \multirow{8}{*}{$(1,2)$} & 1 & 214.98 & 130.69 & 2.31 & 6.80 & 112.35 & 16.99 \\
\hline & 2 & 104.82 & 46.00 & -0.09 & 4.58 & 115.05 & 17.09 \\
\hline & 3 & 39.88 & 10.63 & -3.22 & 1.67 & 118.49 & 17.01 \\
\hline & 4 & 9.40 & 1.16 & -7.93 & -2.71 & 123.21 & 17.33 \\
\hline & 5 & 42.46 & 0.00 & -3.17 & -100000000 & 119.34 & 14.37 \\
\hline & 6 & 33.91 & 7.88 & -3.56 & 1.36 & 118.85 & 16.99 \\
\hline & 7 & 12.08 & 1.64 & -6.88 & -1.71 & 122.29 & 16.91 \\
\hline & 8 & 4.47 & 0.00 & -11.83 & -100000000 & 127.01 & 17.23 \\
\hline \multirow{6}{*}{$(1,3)$} & 9 & 108.93 & 59.66 & 3.40 & 9.92 & 172.01 & 25.94 \\
\hline & 10 & 86.30 & 39.07 & 2.81 & 9.14 & 167.13 & 38.38 \\
\hline & 11 & 26.25 & 6.00 & -1.04 & 5.48 & 171.85 & 38.53 \\
\hline & 12 & 76.81 & 32.73 & 2.38 & 8.74 & 167.98 & 37.29 \\
\hline & 13 & 35.37 & 11.04 & -0.27 & 6.21 & 170.93 & 38.34 \\
\hline & 14 & 12.84 & 0.00 & -4.20 & -100000000 & 175.65 & 38.48 \\
\hline \multirow{5}{*}{$(4,2)$} & 15 & 111.48 & 61.88 & 2.94 & 8.64 & 143.81 & 22.27 \\
\hline & 16 & 94.03 & 48.33 & 2.58 & 8.13 & 140.08 & 31.31 \\
\hline & 17 & 38.22 & 11.91 & -0.22 & 5.47 & 143.51 & 31.26 \\
\hline & 18 & 10.76 & 1.63 & -4.28 & 1.61 & 148.23 & 31.44 \\
\hline & 19 & 34.26 & 0.00 & -0.57 & -100000000 & 144.36 & 29.91 \\
\hline \multirow{6}{*}{$(4,3)$} & 20 & 99.20 & 58.35 & 3.98 & 11.62 & 196.48 & 31.02 \\
\hline & 21 & 87.67 & 45.01 & 3.69 & 11.17 & 192.45 & 40.99 \\
\hline & 22 & 46.01 & 22.99 & 1.56 & 9.25 & 197.04 & 36.89 \\
\hline & 23 & 52.78 & 18.02 & 1.77 & 9.23 & 192.16 & 46.49 \\
\hline & 24 & 16.02 & 4.13 & -2.00 & 5.64 & 196.88 & 46.61 \\
\hline & 25 & 44.82 & 0.00 & 1.25 & -100000000 & 193.01 & 45.59 \\
\hline
\end{tabular}


Yan D, Guo J. A Multiclass Cumulative Prospect Theory-Based Stochastic User Equilibrium Model with Path Constraints in...

Table 4 - The network equilibrium solutions calculated by the EUT-SUE model with distance limit

\begin{tabular}{|c|c|c|c|c|c|c|}
\hline O-D & Path & GV flow & BEV flow & Mean path time (GV) & Mean path time (BEV) & Path time st. dev. \\
\hline \multirow{8}{*}{$(1,2)$} & 1 & 213.79 & 137.61 & 113.16 & 113.16 & 17.05 \\
\hline & 2 & 100.57 & 41.18 & 115.71 & 115.71 & 17.21 \\
\hline & 3 & 42.50 & 10.01 & 118.45 & 118.45 & 17.06 \\
\hline & 4 & 11.55 & 1.16 & 122.67 & 122.67 & 17.34 \\
\hline & 5 & 40.80 & 0.00 & 118.66 & 100000000.00 & 14.30 \\
\hline & 6 & 33.20 & 6.47 & 119.16 & 119.16 & 17.02 \\
\hline & 7 & 14.13 & 1.57 & 121.90 & 121.90 & 16.86 \\
\hline & 8 & 5.46 & 0.00 & 126.12 & 100000000.00 & 17.15 \\
\hline \multirow{6}{*}{$(1,3)$} & 9 & 103.51 & 52.12 & 168.83 & 168.83 & 25.18 \\
\hline & 10 & 89.62 & 42.95 & 169.09 & 169.09 & 39.24 \\
\hline & 11 & 24.60 & 5.03 & 173.30 & 173.30 & 39.36 \\
\hline & 12 & 85.18 & 39.46 & 169.29 & 169.29 & 38.12 \\
\hline & 13 & 32.67 & 8.94 & 172.54 & 172.54 & 39.15 \\
\hline & 14 & 10.92 & 0.00 & 176.76 & 100000000.00 & 39.28 \\
\hline \multirow{5}{*}{$(4,2)$} & 15 & 100.53 & 55.78 & 141.74 & 141.74 & 21.93 \\
\hline & 16 & 97.84 & 53.02 & 141.94 & 141.94 & 31.77 \\
\hline & 17 & 41.04 & 13.39 & 144.48 & 144.48 & 31.69 \\
\hline & 18 & 11.17 & 1.56 & 148.69 & 148.69 & 31.84 \\
\hline & 19 & 38.17 & 0.00 & 144.68 & 100000000.00 & 30.28 \\
\hline \multirow{6}{*}{$(4,3)$} & 20 & 107.50 & 62.21 & 192.11 & 192.11 & 30.17 \\
\hline & 21 & 90.95 & 47.75 & 192.58 & 192.58 & 41.59 \\
\hline & 22 & 45.16 & 15.81 & 195.11 & 195.11 & 36.70 \\
\hline & 23 & 47.02 & 20.02 & 194.86 & 194.86 & 47.46 \\
\hline & 24 & 12.52 & 2.71 & 199.33 & 199.33 & 47.56 \\
\hline & 25 & 43.35 & 0.00 & 195.32 & 100000000.00 & 46.54 \\
\hline
\end{tabular}

assigned by the EUT-SUE model with distance limit. This may be because these two models have different behavioural assumptions. The improved CPT-SUE model assumes that travellers are bounded rational when choosing a path. In other words, when travellers choose a path, they will first judge the gains and losses based on the reference point, and then they will combine the gains and losses with subjective probability weights to obtain the CPVs of different paths. Finally, the travellers choose the path based on the CPV of each path. In contrast, the EUT-SUE model with distance limit assumes that all travellers are perfectly rational when choosing a path. They choose a path based on mean path time without a series of psychological processes required by the cumulative prospect theory.

On the other hand, in Table 3, some travellers choose paths with high mean travel time but low travel time standard deviation. More specifically, the mean travel time on path 5 is larger than that of path 3 or path 6 , but its standard deviation of path travel time is smaller than these two paths. Some travellers who require high travel time reliability will choose paths with less travel time fluctuation, so that the flow on path 5 is higher than that of these two routes. Similar situation also occurs in other OD pairs. In Table 4, all travellers choose a path with a low mean travel time without considering travel time standard deviation. In other words, the results of the path flow assigned by the proposed model show that travellers are bounded rational when choosing a path, while the results of the path flow assigned by the EUT-SUE model with distance limit indicate that travellers are perfectly rational when choosing a path. Therefore, it can be concluded that the proposed model can deal with the perfectly rational issue. 


\subsection{Influence of distance limit parameter on path flows}

In order to verify the capability of the proposed model to deal with range anxiety issue, we analyse the influence of distance limit on path flows of GV and BEV. More specifically, for all integer values of the path length (see Table 2), we studied the influence of integer value of the distance limit (i.e., 27 to 45 ) on the path flows of GV and BEV, as shown in Figure 2.
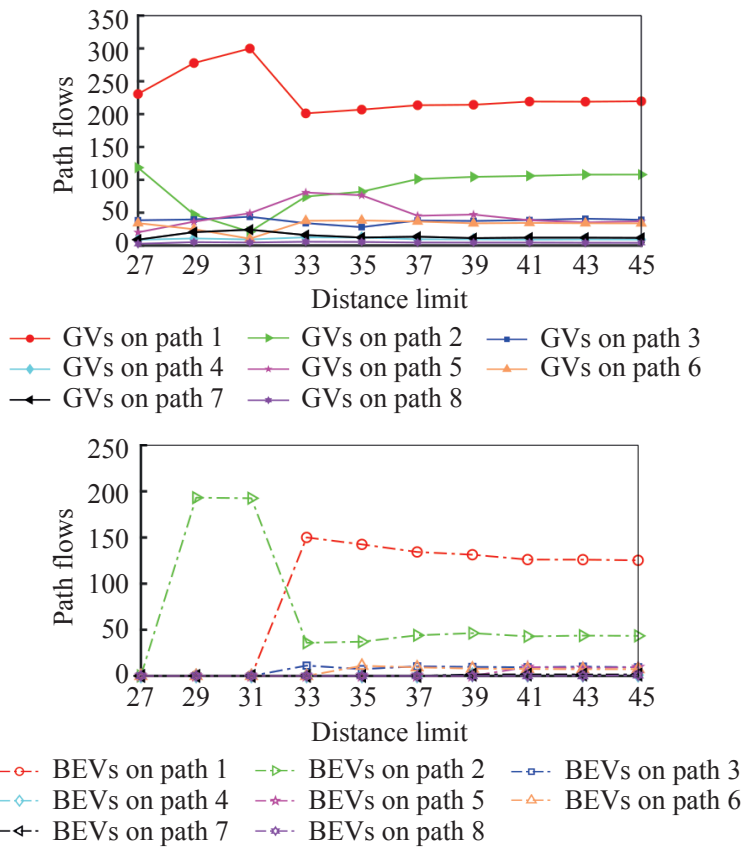

a) O-D pair (1, 2)
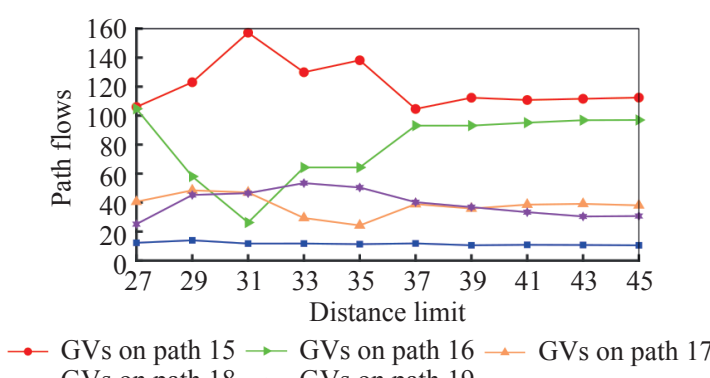

$\rightarrow$ GVs on path $18 \rightarrow$ GVs on path 19

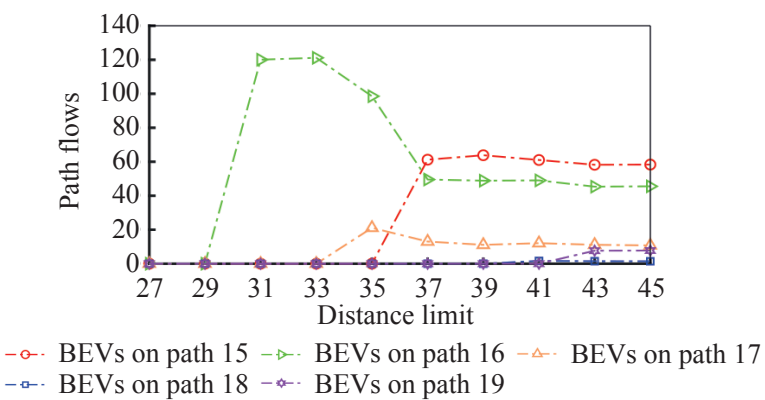

c) O-D pair $(4,2)$
First, it can be seen that each path carries the GV flow under different distance limits because the driving range of the $\mathrm{GV}$ is not subject to distance limit. In contrast, not every path carries BEV flow under different distance limits, for example, when the distance limit is equal to 27 , the BEV flow on all paths is zero. This is because the distance limit significantly affects the driving range of the BEV.

Second, it can be observed that path flows of the GV and the BEV mainly focus on two paths (e.g. path 1 and path 2). Other paths carry very
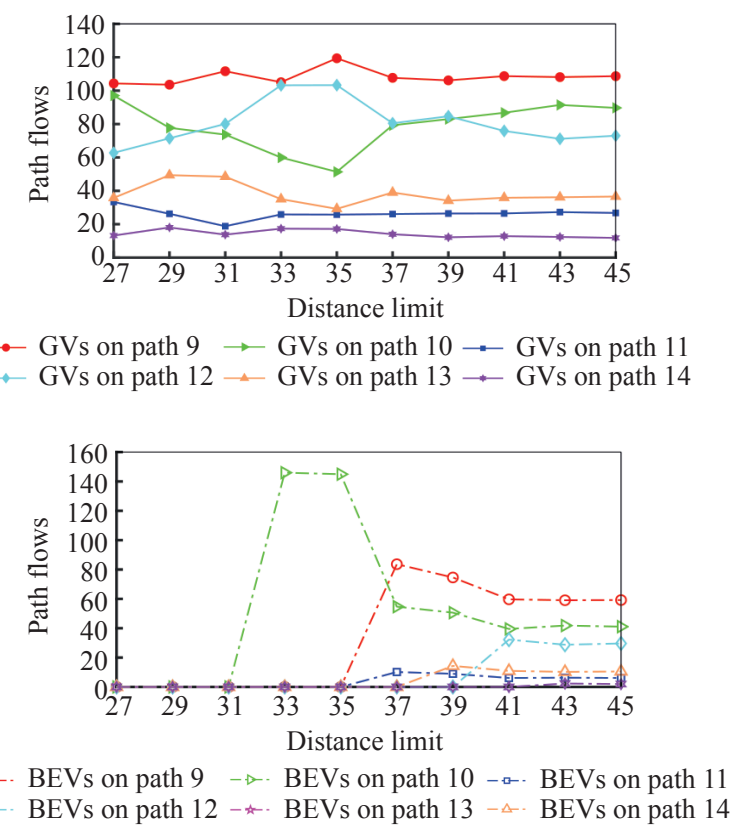

b) $O$-D pair $(1,3)$
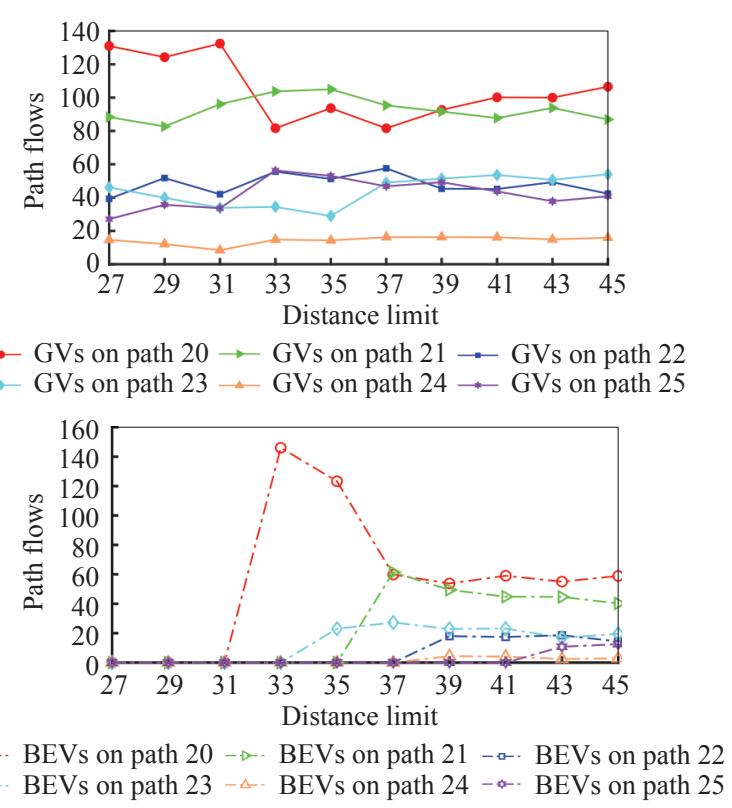

d) O-D pair (4, 3)

Figure 2 - Variations of path flows of $G V$ and BEV with the distance limit 
little flows, especially the BEV flow. This may be because these two paths have less travel time and higher travel time reliability than other paths. In this way, the CPVs of these two paths will be greater. Therefore, more flow will be assigned to these two paths.

Third, when the distance limit becomes tighter, the variations of the GV flow and the BEV flow with distance limit are opposite on some paths (e.g. path 1 , path 9 , and path 15). This may be because route choice behaviour of BEV users is strongly restricted by distance limit, so they tend to choose paths that are not subject to distance limit. In this case, these paths chosen by BEV users will become oversaturated. Further, this will cause an important decline in the capacity of the links contained in those paths, which in turn will lead to a substantial increase in travel time and a significant reduction in travel time reliability. In this way, GV users prefer to use those unsaturated paths to reduce their travel time. Therefore, on some paths, the changes of GV flow and BEV flow with distance limit will be opposite.

Finally, when the distance limit becomes looser, the variations of the GV flow and the BEV flow with the distance limit are slightly similar on some paths (e.g. path 2, path 12, and path 21). This may be because distance limit gradually loses the restriction on BEV users. In this case, there is no difference between BEV users and GV users when choosing a path, that is, they tend to choose a path with a larger CPV.

\subsection{Generality analysis of the improved CPT-SUE model}

In order to verify the generality of the proposed improved CPT-SUE model, we run this model and the associated algorithm on a small network. The small network consists of 1 O-D pair and 5 links, as shown in Figure 3. The O-D demand is 1000 . The distance limit of BEV is set to $D_{2}=12$. The other parameters are the same as those in the Nguyen-Dupuis network.

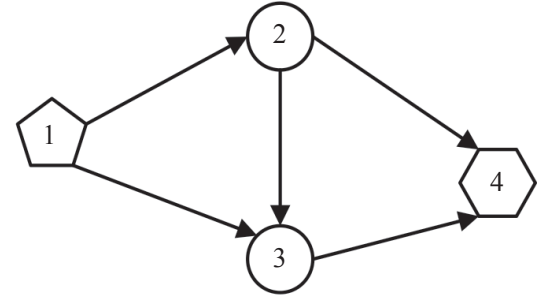

Origin node $\bigcirc$ Destination node $\bigcirc$ Ordinary node

Figure 3 - Small network

The free-flow travel time, i.e., $t_{a}^{0}$ and capacity of each link, i.e., $\bar{c}_{a}$ are shown in Table 5, and the path composition and path length are exhibited in Table 6 (The values in Table 5 and Table 6 are adopted from $\mathrm{Li}$ and Lang [45]). In addition, the worst-degraded coefficient $\theta_{a}$ for each link is also shown in Table 5 .

The improved CPT-SUE model and the EUTSUE model with distance limit are run on the small network respectively, and then the network equilibrium solutions are obtained as shown in Table 7 and Table 8. In addition, for this small network, we also studied the influence of distance limit on path flows of the GV and the BEV, as shown in Figure 4.

Table 5 - Link characteristics

\begin{tabular}{||c|c|c|c|c||}
\hline \hline No. & Link & $t_{a}^{0}$ & $\bar{c}_{a}$ & $\theta_{a}$ \\
\hline \hline 1 & $1-2$ & 5 & 600 & 0.6 \\
\hline 2 & $1-3$ & 6 & 500 & 0.6 \\
\hline 3 & $2-3$ & 7 & 600 & 0.8 \\
\hline 4 & $2-4$ & 8 & 500 & 0.7 \\
\hline 5 & $3-4$ & 3 & 700 & 0.7 \\
\hline
\end{tabular}

Table 6 - Path composition and length

\begin{tabular}{|c|c|c||}
\hline Path & Node sequence & Path length \\
\hline \hline 1 & $1-2-4$ & 13 \\
\hline 2 & $1-2-3-4$ & 14 \\
\hline 3 & $1-3-4$ & 9 \\
\hline
\end{tabular}

Table 7 - The network equilibrium solutions calculated by the improved CPT-SUE model

\begin{tabular}{||c|c|c|c|c|c|c|c||}
\hline \hline O-D & Path & GV flow & BEV flow & CPV (GV) & CPV (BEV) & Mean path time & Path time st. dev. \\
\hline \hline \multirow{3}{*}{$(1,2)$} & 1 & 311.77 & 0 & 0.04 & -100000000 & 14.12 & 0.47 \\
\cline { 2 - 8 } & 2 & 140.74 & 0 & -2.57 & -100000000 & 15.63 & 0.57 \\
\cline { 2 - 8 } & 3 & 247.48 & 300 & -0.72 & -0.56 & 13.81 & 2.36 \\
\hline
\end{tabular}


Yan D, Guo J. A Multiclass Cumulative Prospect Theory-Based Stochastic User Equilibrium Model with Path Constraints in...

Table 8 - The network equilibrium solutions calculated by the EUT-SUE model with distance limit

\begin{tabular}{|c|c|c|c|c|c|c||}
\hline \hline O-D & Path & GV flow & BEV flow & Mean path time (GV) & Mean path time (BEV) & Path time st. dev. \\
\hline \hline \multirow{3}{*}{$(1,4)$} & 1 & 281.75 & 0 & 13.93 & 100000000 & 0.42 \\
\cline { 2 - 7 } & 2 & 161.76 & 0 & 15.75 & 100000000 & 0.60 \\
\cline { 2 - 7 } & 3 & 256.48 & 300 & 14.24 & 14.24 & 2.53 \\
\hline
\end{tabular}
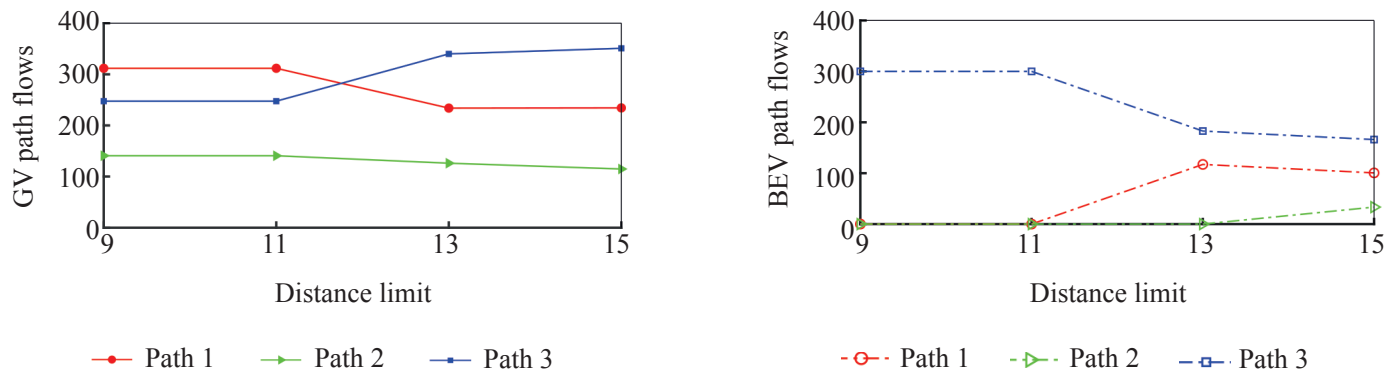

Figure 4 - Variations of path flows of $G V$ and BEV with the distance limit

In Table 7 and Table 8, it can be seen that the traveller's route choice behaviour is the same as that of Table 3 and Table 4. More specifically, the network equilibrium solutions calculated by the improved CPT-SUE model show that some travellers choose paths with high mean but low standard deviation, such as path 1 compared to path 3 . The network equilibrium solutions calculated by the EUT-SUE model with distance limit indicate that all travellers choose a path with a low mean without considering standard deviation. In addition, it can be seen that the change trend of path flows of GV and BEV with distance limit is similar to that of Nguyen-Dupuis network. For example, the variations of the GV flow and the BEV flow with distance limit are opposite on some paths. Therefore, it can be concluded that the proposed improved CPT-SUE model has general applicability.

\section{CONCLUSIONS}

With the increasing adoption of BEVs in road networks, range anxiety is inevitably considered in the process of traffic assignment modelling. In addition, perfectly rational issue is also to be handled for traffic assignment models to provide a better descriptive paradigm for individuals' decision making under risk or uncertainty. In order to deal with these two issues, this paper improves the CPT-SUE model in a degradable transport network through incorporating the constraints of multiple user classes and distance limit, for which, an equivalent variational inequality model and associated MSA based solution are provided, and the existence and equivalence of model solution are also proved.

The numerical test is conducted on the wellknown Nguyen-Dupuis network and small network to compare path flows of GV and BEV calculated by the improved CPT-SUE model and EUT-SUE model with distance limit, and to perform analysis of the influence of distance limit on path flows of $\mathrm{GV}$ and BEV. The network equilibrium solutions calculated by the improved CPT-SUE model indicate that the travellers are boundedly rational when making route choice decisions. More specifically, some travellers choose paths with high mean but low standard deviation. In addition, distance limit has a significant impact on the assignment results of path flows of GV and BEV, that is, the loose or tight distance limit determines that the GV flow and the BEV flow have opposite or similar trends. Therefore, it can be concluded that the improved CPTSUE model can solve the range anxiety issue and perfectly rational issue jointly.

In the future, the improved CPT-SUE model can be tested on a larger network to find a balance between the computational tractability and model complexity. In addition, elastic demand instead of fixed demand can also be incorporated to further improve the proposed model.

\section{ACKNOWLEDGMENT}

This research is supported by the Postgraduate Research \& Practice Innovation Program of Jiangsu Province (No. KYLX16_0271). 
问冬梅, 博士研究生 ${ }^{1}$

(通讯作者)

电子邮箱: ydm 1988@163.com

郭建华, 博士 ${ }^{1}$

E-mail:jg2nh@yahoo.com

1 东南大学智能运输系统研究中心

中国南京市江宁区东南大学路2号

\section{路径约束下基于累积前景理论的多用户降级路 网 $\boldsymbol{S U E}$ 模型}

\section{摘要}

在纯电动汽车 $(B E V)$ 的交通分配中，有限的 驾驶范围以及电池充电站的不可用或不充足导致了 范围焦虑问题。此外，出行者路径选择决策时，基 于期望效用理论的随机用户平衡 (EUT-SUE) 模型 具有完全理性问题。为了解决这两个问题，本文通 过考虑多用户类和距离限制的约束, 改进了降级路 网中基于累积前景理论的随机用户平衡 (CPT-SUE) 模型。在该降级路网中, 路段通行能力下降导致 出行者出行时间的随机波动。对于改进的 CPT-SUE 模型，我们提供了等价的变分不等式 $(V I)$ 模型和相 继平均法 $(M S A)$ 的算法。我们测试并比较了改进的 $C P T-S U E$ 模型和具有距离限制的 EUT-SUE模型, 结 果表明, 改进的CPT-SUE模型可以同时解决范围焦 虑问题和完全理性问题。

\section{关键词}

随机用户平衡; 累积前景理论; 期望效用理论; 距离限制; 变分不等式; 相继平均法

\section{REFERENCES}

[1] Boston Consulting Group. The comeback of the electric car?; 2009.

[2] Addison J. National academies predicts 13 to 40 million plug-ins by 2030; 2012. Available from:http://www. cleanfleetreport.com/plug-in-hybrids/nationalacademies-40-million-plug-ins/

[3] Pearre NS, Kempton W, Guensler RL, Elango VV. Electric vehicles: how much range is required for a day's driving? Transportation Research Part C: Emerging Technologies. 2011;19(6): 1171-1184. DOI: 10.1016/j. trc.2010.12.010

[4] Morrow K, Karner D, Francfort J. Plug-in hybrid electric vehicle charging infrastructure review. U.S', Department of Energy Vehicle Technologies Program, Washington DC; 2008.

[5] Dong J, Liu CZ, Lin ZH. Charging infrastructure planning for promoting battery electric vehicles: An activity-based approach using multiday travel data. Transportation Research Part C: Emerging Technologies. 2014;38: 44-55. DOI: $10.1016 /$ j.trc.2013.11.001

[6] Xie C, Wu X, Boyles S. Traffic equilibrium with a continuously distributed bound on travel weights: The rise of range anxiety and mental account. Annals Operations Research. 2018;273(1-2): 279-310. DOI: $10.1007 /$ s10479-018-2990-0
[7] Jiang N, Xie C, Waller ST. Path-constrained traffic assignment: Model and algorithm. Transportation Research Record. 2012;2283: 25-33. DOI: 10.3141/2283-03

[8] He F, Yin YF, Lawphongpanich S. Network equilibrium models with battery electric vehicles. Transportation Research Part B: Methodological. 2014;67: 306-319. DOI: 10.1016/j.trb.2014.05.010

[9] Jiang N, Xie C. Computing and analyzing mixed equilibrium network flows with gasoline and electric vehicles. Computer Aided Civil and Infrastructure Engineering. 2014;29(8): 626-641. DOI: 10.1111/mice.12082

[10] Xu M, Meng Q, Liu K. Network user equilibrium problems for the mixed battery electric vehicles and gasoline vehicles subject to battery swapping stations and road grade constraints. Transportation Research Part B: Methodological. 2017;99: 138-166. DOI: 10.1016/ j.trb.2017.01.009

[11] Liu H, Wang DZ. Locating multiple types of charging facilities for battery electric vehicles. Transportation Research Part B: Methodological. 2017;103: 30-55. DOI: 10.1016/j.trb.2017.01.005

[12] Miralinaghi $M$, et al. Refueling station location problem with traffic deviation considering route choice and demand uncertainty. International Journal of Hydrogen Energy. 2017;42(5): 3335-3351. DOI: 10.1016/ j.ijhydene.2016.12.137

[13] Jing W, An K, Ramezani M, Kim I. Location design of electric vehicle charging facilities: A path distance constrained stochastic user equilibrium approach. Journal of Advanced Transportation. 2017;1-15. DOI: 10.1155/2017/4252946

[14] Maher M. Algorithms for logit-based stochastic user equilibrium assignment. Transportation Research Part B: Methodological. 1998;32(8): 539-549. DOI: 10.1016/ S0191-2615(98)00015-0

[15] Yang Y, Yan YS, Hu ZA, Xie P. Algorithm research for capacity-constrained stochastic traffic assignment model. International Journal of Information \& Communication Technology. 2014;6(3/4): 241-250. DOI: 10.1504/ IJICT.2014.063214

[16] Zhou Z, Chen A, Bekhor S. C-logit stochastic user equilibrium model: formulations and solution algorithm. Transportmetrica. 2012;8(1): 17-41. DOI: $10.1080 / 18128600903489629$

[17] Bekhor S, Toledo T, Reznikova L. A path-based algorithm for the cross-nested logit stochastic user equilibrium traffic assignment. Computer Aided Civil \& Infrastructure Engineering. 2010;24(1): 15-25. DOI: 10.1111/j.14678667.2008.00563.x

[18] Von Neumann J, Morgenstern O. Theory of games and economic behaviour (commemorative edition). Princeton University Press; 1994.

[19] Jiang N, Xie C, Duthie JC, Waller ST. A network equilibrium analysis on destination, route and parking choices with mixed gasoline and electric vehicular flows. Euro Journal of Transportation and Logistics. 2014;3(1): 5592. DOI:10.1007/s13676-013-0021-5

[20] Liu Z, Song Z. Network user equilibrium of battery electric vehicles considering flow-dependent electricity consumption. Transportation Research Part C: Emerging Technologies. 2018;95: 516-544. DOI: 10.1016/ j.trc.2018.07.009 
[21] He F, Wu D, Yin Y, Guan Y. Optimal deployment of public charging stations for plug-in hybrid electric vehicles. Transportation Research Part B: Methodological. 2013;47: 87-101. DOI: 10.1016/j.trb.2012.09.007

[22] Li SY, Huang YX, Mason SJ. A multi-period optimization model for the deployment of public electric vehicle charging stations on network. Transportation Research Part C: Emerging Technologies. 2016;65: 128-143. DOI: 10.1016/j.trc.2016.01.008

[23] Chen Z, Liu W, Yin Y. Deployment of stationary and dynamic charging infrastructure for electric vehicles along traffic corridors. Transportation Research Part C: Emerging Technologies. 2017;77: 185-206. DOI: 10.1016/j.trc.2017.01.021

[24] Zhang X, Rey D, Waller ST, Chen N. Range-constrained traffic assignment with multi-modal recharge for electric vehicles. Networks and Spatial Economics. 2019;19: 633-668. DOI: 10.1007/s11067-019-09454-9

[25] Adler JD, Mirchandani PB. Online routing and battery reservations for electric vehicles with swappable batteries. Transportation Research Part B: Methodological. 2014;70: 285-302. DOI: 10.1016/j.trb.2014.09.005

[26] Liao CS, Lu SH, Shen ZJM. The electric vehicle touring problem. Transportation Research Part B: Methodological. 2016;86: 163-180. DOI: 10.1016/j.trb.2016.02.002

[27] Schneider M, Stenger A, Goeke D. The electric vehicle-routing problem with time windows and recharging stations. Transportation Science. 2014;48: 500-520. DOI: $10.1287 /$ trsc. 2013.0490

[28] Jing WT, Kim I, Ramezani M, Liu ZY. Stochastic traffic assignment of mixed electric vehicle and gasoline vehicle flow with path distance constraints. Transportation Research Procedia. 2017;21: 65-78. DOI: 10.1016/j.trpro. 2017.03.078

[29] Ma J, Cheng L, Li DW, Tu Q. Stochastic electric vehicle network considering environmental costs. Sustainability. 2018;10(8): 1-16. DOI: 10.3390/su10082888

[30] Tversky A, Kahneman D. Advances in prospect theory: Cumulative representation of uncertainty. Journal of Risk and Uncertainty. 1992;5(4): 297-323. DOI: 10.1007/BF00122574

[31] Connors RD, Sumalee A. A network equilibrium model with travelers' perception of stochastic travel times. Transportation Research Part B: Methodological. 2009;43(6): 614-624. DOI: 10.1016/j.trb.2008.12.002

[32] Lou YY, Yin YF, Lawphongpanich S. Robust congestion pricing under boundedly rational user equilibrium. Transportation Research Part B: Methodological. 2010;44(1): 15-28. DOI: 10.1016/j.trb.2009.06.004

[33] Xu HL, Lou YY, Yin YF, Zhou J. A prospect-based user equilibrium model with endogenous reference points and its application in congestion pricing. Transportation Research Part B: Methodological. 2011;45(2): 311-328. DOI: $10.1016 /$ j.trb.2010.09.003

[34] Wang Q, Xu W. A user equilibrium model based on cumulative prospect theory for degradable transport network. Proceedings of the $4^{\text {th }}$ International Joint Conference on Computational Sciences and Optimization; 2011.

[35] Wang W, Sun HJ. Cumulative prospect theory-based user equilibrium model with stochastic perception errors. Journal of Central South University. 2016;23(9): 24652474. DOI: 10.1007/s11771-016-3305-8

[36] Yang JF, Jiang GY. Development of an enhanced route choice based on cumulative prospect theory. Transportation Research Part C: Emerging Technologies. 2014;47(2): 168-178. DOI: 10.1016/j.trc.2014.06.009

[37] Bureau of Public Roads. Traffic assignment manual. U.S. Department of Commerce, Urban Planning Division, Washington D.C.; 1964.

[38] Lo HK, Siu BWY. Degradable transport network: Travel time budget of travellers with heterogeneous risk aversion. Transportation Research Part B: Methodological. 2006;40(9): 792-806. DOI: 10.1016/j.trb.2005.10.003

[39] Kahneman D, Tversky A. Prospect theory: An analysis of decisions under risk. Econometrica. 1979;47 (2): 263291. DOI: $10.2307 / 1914185$

[40] Thaler RH, Tversky A, Kahneman D, Schwartz A. The effect of myopia and loss aversion on risk taking: An experimental test. Quarterly Journal of Economics. 1997;112(2): 647-661. DOI: 10.1162/003355397555226

[41] Camerer CF, Hho TH. Violations of the betweenness axiom and nonlinearity in probability. Journal of Risk and Uncertainty. 1994;8(2): 167-196. DOI: 10.1007/ BF01065371

[42] Prelec D. The probability weighting function. Econometrica. 1998;66(3): 497-527. DOI: 10.2307/2998573

[43] Zhang B, Juan ZC, Lin XX. Stochastic user equilibrium model based on cumulative prospect theory. Journal of Southwest Jiaotong University. 2011;46(5): 868-874. DOI: 10.3969/j.issn.0258-2724.2011.05.026

[44] Sheffi Y. Urban transportation networks: Equilibrium analysis with mathematical programming methods. Englewood Cliffs, New Jersey: Prentice-Hall; 1985. p. 214227.

[45] Li XF, Lang, MX. Multi-class and multi-criteria stochastic user equilibrium model based on generalized nested logit model. Journal of Transportation Systems Engineering and Information Technology. 2014;14: 139-145. DOI: 10.3969/j.issn.1009-6744.2014.04.020 